\title{
Research on the Application of Multi-view Image Matching Point Cloud in Engineering Quantity Calculation of Mining Area
}

\author{
ZheWANG $^{1, \mathbf{a}^{*}}$, XiTun YUAN ${ }^{1}$, PengWei GAO ${ }^{1}$, Lei XING ${ }^{1}$ \\ ${ }^{1}$ School of Surveying Science and Technology, Xi' an University of Science and Technology, Xi' an 710054, China
}

\begin{abstract}
For mines with complex natural terrain conditions, especially for open pit mines with poor slope stability; traditional surveying and mapping techniques are difficult to accurately map engineering quantities and have certain safety hazards. Based on the actual production, this paper systematically studies the key technology of multi-view image matching point cloud in the calculation of mining area engineering quantity, and verifies the feasibility and accuracy of it, and compares it with GPS-RTK, traditional aerial survey and other data acquisition technologies. Comparative analysis shows that multi-view image matching point cloud technology has certain advantages in terms of time, accuracy, cost and security risks. ${ }^{1, \mathbf{a}^{*}}$
\end{abstract}

\section{Introduction}

China has a vast territory and rich mineral resources; the shallow buried deposits usually adopt open-pit mining methods; and the open-pit mining area often involves the calculation of engineering quantities in the process of early construction, mining and production and land reclamation, and is aimed at the engineering quantities of open-pit mining areas. The measurement has the characteristics of separate measurement, continuous measurement, and large engineering quantity ${ }^{[1]}$. It is particularly important to select an efficient, accurate and safe engineering quantity measurement method.

The traditional engineering measurement data is mainly collected by GPS-RTK, total station, threedimensional laser scanning, Lidar, aerial photogrammetry and other methods; among them, GPS-RTK, total station and other field survey data collection methods are more expensive. Large, low efficiency, and low density of sampling points ${ }^{[2]}$; three-dimensional laser scanning, lidar and other data collection methods have good terrain details, but they have the disadvantage of higher production costs ${ }^{[3]}$; aerial photogrammetry is also due to airspace applications and production Disadvantages such as long cycle are rarely applied in actual engineering quantity calculation. Unmanned aerial vehicle (UAV) multi-vision photogrammetry technology is widely used in the surveying and mapping industry due to its flexible operation, and multiple auxiliary products. Therefore, this article applies multi-view image matching point cloud to the calculation of engineering quantity.

This paper systematically introduces the key technology of multi-view image matching point cloud, analyzes the feasibility and accuracy of this technology, and compares it with other data acquisition technologies.

\section{Realization of multi-view image matching point cloud in engineering quantity calculation}

First, pierce the image control points in the multi-view image, extract the feature points in the multi-view image and perform SIFT rough matching based on the nearest neighbor distance ratio, and then eliminate the mismatched points to complete the matching of the multiview image. Secondly, the beam method area network adjustment is performed on the multi-view image to obtain the external orientation elements of the multi-view image. Third, the PMVS algorithm is used to perform dense matching of multi-view images to obtain DSM point clouds. Finally, the Delaunay triangulation network is constructed based on the DSM point cloud and the DTM method is used for engineering quantity calculation. The specific implementation process is shown in Figure 1.

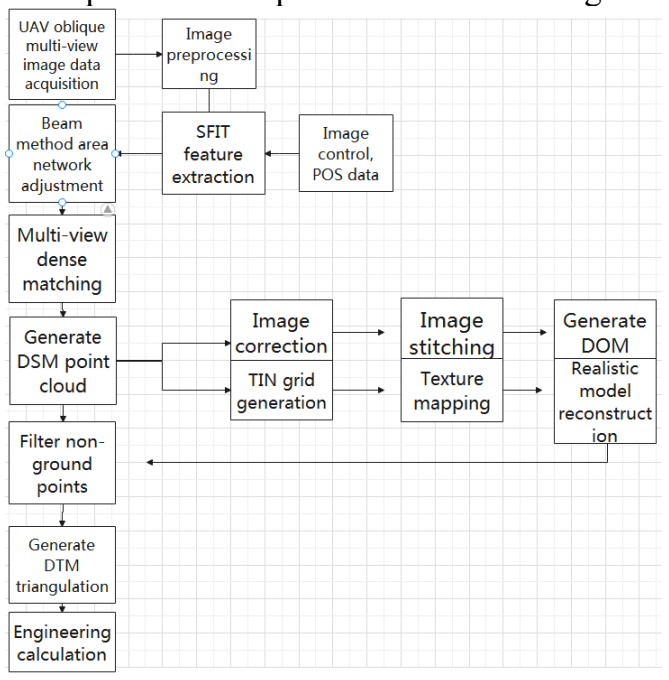

Fig1. Implementation flowchart 


\subsection{The key technology of multi-view image matching point cloud}

SIFT operator feature point extraction machine and rough matching; using SIFT operator to extract feature points and rough matching of multi-view images, the steps are: use different Gaussian kernel functions to perform convolution operations on multi-view images to generate images with different definitions, Which is to produce image Gaussian pyramid; image scale space $\mathrm{L}(\mathrm{x}, \mathrm{y}, \sigma)$ is obtained by convolution operation of image I $(x, y)$ and Gaussian function $\mathrm{G}(\mathrm{x}, \mathrm{y}, \sigma)^{[4]}$, as in equation (1) .

$$
\mathrm{L}(\mathrm{x}, \mathrm{y}, \sigma)=\mathrm{I}(\mathrm{x}, \mathrm{y}) * \mathrm{G}(\mathrm{x}, \mathrm{y}, \sigma)
$$

In the formula, $G(x, y, \sigma)=\frac{1}{2 \Pi \sigma^{2}} e^{\frac{x^{2}+y^{2}}{2 \sigma^{2}}}, \sigma$ is the scale parameter, ${ }^{*}$ is the convolution operation.

The generated adjacent image scale space is subtracted to obtain the differential Gaussian pyramid (DoG). This process completes the construction of the scale space, and then performs scale space extreme value detection; performs key point (feature point) positioning; key feature point description, and finally Normalize the length of the feature vector ${ }^{[5]}$.

Light speed regional network adjustment; beam method regional network adjustment takes the light corresponding to the multi-view image as the basic unit, the collinear equation as the mathematical model, and the common intersection coordinates and control point coordinates of adjacent images as the adjustment conditions , List the error equations of control points and encryption points ${ }^{[6]-[7]}$, such as (2); incorporate the multiview images of the entire survey area into the known object coordinates, and calculate each image The external orientation elements of the multi-view image and the object coordinates of all encrypted points.

$$
\mathrm{V}=\left[\begin{array}{ll}
\mathrm{A} & \mathrm{B}
\end{array}\right]\left[\begin{array}{l}
\mathrm{t} \\
\mathrm{X}
\end{array}\right]-\mathrm{L}
$$

Where: $\mathrm{V}$ matrix is the correction matrix of the image point coordinate error;

Matrix A and B are the coordinate correction coefficient matrix corresponding to the control point and the connection point;

The matrix of $\mathrm{t}$ and $\mathrm{X}$ is a matrix of unknowns (external orientation element, internal orientation element, three-dimensional coordinates of the encrypted point, distortion parameter);

The $\mathrm{L}$ matrix is a constant term matrix of the difference between the observed value of the image point and the calculated result.

Multi-view image dense matching technology; the PMVS algorithm is used for dense matching of multi-view images to obtain high-density 3D point cloud data. POS information can match a large number of dense point cloud data with RGB information, thereby constructing a fine real 3D model. This algorithm is a simple, efficient, and cost-effective matching method ${ }^{[8]}$. The basic process of PMVS algorithm includes (1) initial feature matching; (2) patch diffusion; (3) patch filtering.

\subsection{Engineering quantity calculation}

The engineering quantity calculation adopts the DTM
(Digital Terrain Model, digital terrain model) method. The DTM method uses two phases of DTM data to generate ground point cloud coordinates $(\mathrm{X}, \mathrm{Y}, \mathrm{Z})$ before and after the excavation and filling of the measured engineering quantity area. According to the irregular triangular network files of the ground surface before and after the excavation and filling, the irregular triangular prism is used as the calculation unit to calculate the excavation and filling works according to the triangulation file, and then the excavation and filling works in the delimited area are calculated iteratively ${ }^{[9]}$.

\section{Test and result analysis}

This experiment was carried out in an open-pit mining area. Field multi-view image acquisition, image control measurement and multi-view image in-house processing were carried out, and the progress of the point cloud of the result of multi-view image matching was analyzed.

\subsection{Multi-view image acquisition}

This multi-view image data is obtained by using the DJI UAV (UAV) equipped with a multi-view camera pan/tilt system to perform multi-view photography of targets in the mining area.

Aeroflight design; high overlap, the heading and side overlap are set at more than $80 \%$, to improve the quality of the generated multi-view image matching point cloud, and to solve the problem of occlusion in the survey area.

Layout of image control points and check points; dense image control, in order to ensure the absolute accuracy of multi-view image matching point clouds, dense image control should be adopted, and the layout method should be evenly distributed around the pit and at the bottom of the pit

\subsection{Multi-view image processing}

According to the implementation method of the abovementioned multi-view image matching point cloud in the engineering quantity calculation, the multi-view image data collected by the field industry is first subjected to uniform light and color preprocessing; the POS data is checked, the images with bad postures are eliminated, and the off course is eliminated Of the image. Then import the pre-processed multi-view images into Smart3D for air three processing, dense matching to generate DSM point clouds, and then artificially intervene in point cloud filtering to filter out non-ground points, and then build a Delaunay triangulation .

Orthoimages can be generated by DSM automatic filtering, image correction, and image stitching (as shown in Figure 2); TIN triangular meshes can also be constructed from DSM point clouds, and then textures are automatically mapped to generate 3D real scene models, as shown in Figure 3. 


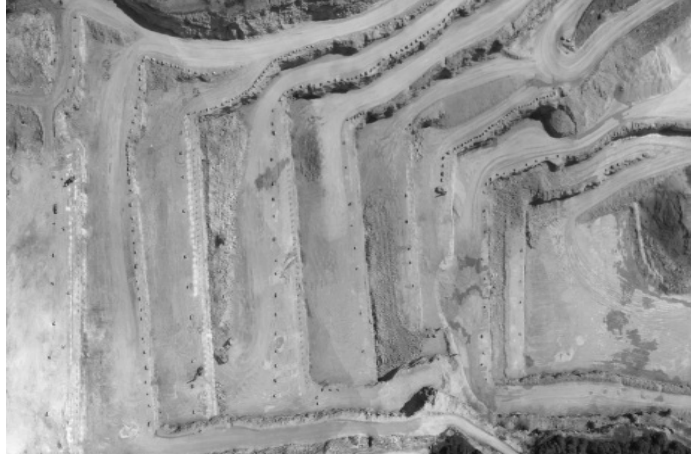

Fig2. Orthophoto map

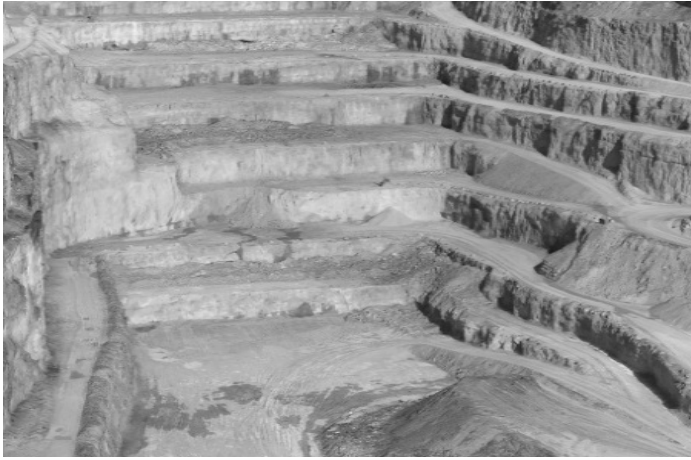

Fig3.3D real scene model

\subsection{Result analysis}

The aerial triangulation is carried out using GPS/IMU assisted aerial triangulation technology. The control point encryption accuracy is shown in Table 1:

Table1. Table 1 Statistical table of residual values of control points Unit: $\mathrm{m}$

\begin{tabular}{cccc}
\hline $\begin{array}{c}\text { Control } \\
\text { point }\end{array}$ & $\mathrm{V}_{\mathrm{X}} / \mathrm{m}$ & $\mathrm{V}_{\mathrm{Y}} / \mathrm{m}$ & $\mathrm{V}_{\mathrm{Z}} / \mathrm{m}$ \\
\hline $\mathrm{GCP001}$ & 0.026 & 0.048 & -0.081 \\
$\mathrm{GCP002}$ & 0.012 & -0.071 & -0.039 \\
$\mathrm{GCP003}$ & -0.041 & 0.048 & 0.054 \\
$\mathrm{GCP004}$ & 0.058 & -0.027 & 0.062 \\
$\mathrm{GCP005}$ & 0.063 & -0.024 & 0.047 \\
$\mathrm{GCP006}$ & -0.045 & 0.019 & -0.038 \\
$\mathrm{GCP007}$ & 0.065 & 0.037 & 0.046 \\
$\mathrm{GCP008}$ & 0.013 & -0.028 & -0.060 \\
$\mathrm{GCP009}$ & 0.047 & 0.046 & 0.098 \\
\hline
\end{tabular}

It can be seen from the above table that the air-three encryption accuracy of this test area is good, which can fully meet the accuracy requirements of engineering quantity measurement.

In this experiment, the elevation was checked by using the fragmented points obtained by field measurements. The accuracy statistics table is shown in Table 2:

Table2. Elevation residual value statistics table of checkpoints in detail Unit: $m$

\begin{tabular}{cc|cc}
\hline $\begin{array}{c}\text { Point } \\
\text { number }\end{array}$ & $\mathrm{V}_{\mathrm{Z}} / \mathrm{m}$ & $\begin{array}{c}\text { Point } \\
\text { number }\end{array}$ & $\mathrm{V}_{\mathrm{Z}} / \mathrm{m}$ \\
\hline $\mathrm{P} 001$ & -0.023 & P006 & 0.082
\end{tabular}

\begin{tabular}{ll|ll} 
P002 & 0.096 & P007 & 0.076 \\
P003 & 0.063 & P008 & -0.049 \\
P004 & 0.025 & P009 & -0.035 \\
P005 & -0.017 & P010 & 0.045 \\
\hline
\end{tabular}

From the above table, the maximum residual value of the checkpoint elevation obtained through field measurements is $9.6 \mathrm{~cm}$, and it can be calculated that the error of the checkpoint elevation is $6 \mathrm{~cm}$, which meets the accuracy requirements of the above DTM production.

Since only one flight data collection was performed in this test, the engineering quantity can only be measured at a given elevation, and the engineering quantity cannot be measured by subtracting the two-phase data. However, the key to the DTM triangulation method is to obtain highprecision DTM, so this experiment can still show that the multi-view image matching point cloud is feasible and the accuracy meets the requirements.

\section{Comparative analysis with other data acquisition technologies}

This paper uses GPS-RTK, traditional aerial survey and multi-view image matching point cloud technology to collect and process data in the above areas, and analyzes the time, accuracy, cost and safety hazards of each technology; the comparative analysis is shown in Table 3. Shown.

Table3. Comparison and analysis table of each technology

\begin{tabular}{|c|c|c|c|}
\hline & $\begin{array}{l}\text { GPS- } \\
\text { RTK }\end{array}$ & $\begin{array}{c}\text { Traditional } \\
\text { aerial survey }\end{array}$ & $\begin{array}{l}\text { Multi-view } \\
\text { image } \\
\text { matching } \\
\text { point cloud }\end{array}$ \\
\hline time & general & Longer & Shorter \\
\hline Precision & general & Poor & better \\
\hline cost & general & Higher & Lower \\
\hline $\begin{array}{l}\text { Security } \\
\text { risks }\end{array}$ & Larger & Smaller & Smaller \\
\hline
\end{tabular}

Accuracy: From the analysis of point accuracy alone, GPS-RTK technology is used to collect point data with the highest accuracy, and the point accuracy of multi-view image matching point cloud technology and traditional aerial survey technology decreases sequentially; but from the analysis of detailed terrain performance, multi-view The detail of image matching point cloud technology is better than GPS-RTK and traditional aerial survey technology; for engineering quantity measurement, the collected data not only requires high point position accuracy, but also requires good terrain details of the collected data.

In terms of cost: Compared with GPS-RTK and traditional aerial survey technology that require investment in professional equipment, multi-view image matching point cloud technology only needs to be invested in consumer drones, which greatly reduces the cost.

In terms of potential safety hazards, the use of GPSRTK technology in mining areas with complex terrain 
conditions cannot guarantee the safety of technical personnel, and their safety hazards are relatively high. However, multi-view image matching point clouds and traditional aerial survey technology only need to be in flat areas. Deploy measurement and image control, so that data can be collected, and potential safety hazards are greatly reduced.

According to the summary, compared with GPS-RTK and traditional aerial survey technology, multi-view image matching point cloud technology has certain advantages in terms of time, accuracy, cost and safety hazards for engineering quantity measurement.

\section{Conclusion}

The use of multi-view image matching point cloud technology for mining area engineering quantity measurement; its accuracy is good, and the accuracy of control points and inspection points meets the engineering quantity measurement requirements, which proves the application of multi-view image matching point cloud technology to mining area engineering quantities. The calculation is completely feasible.

Compared with GPS-RTK and traditional aerial survey technology, the use of multi-view image matching point cloud technology for mining engineering quantity calculation is not only flexible, but also has certain advantages in terms of time, accuracy, cost and safety hazards. Promotion and application in actual production operations.

There are many subsidiary products of multi-view image matching point cloud technology, which can be applied to smart mines, mining area planning and construction, mining area dynamic monitoring, land reclamation, and many other projects. This is also the content of this article to be further studied in the future.

\section{References}

1. Li Xiuchun, Xin Zhi, Cai Qingfei, et al. Discussion on calculation of earth-rock stripping engineering quantity in open-pit coal mine[J]. Surface Mining Technology, 2017(01): 32-36.

2. Liu Jianting, Liu Jianpo. Research on application of earthwork calculation in open-pit mine based on GNSS and total station[J]. Mine Survey, 2016, 44(4): 44-48.

3. Li Bin, Ran Lei, Cheng Chengqi. Research on the application of three-dimensional laser scanning technology in earthwork engineering [J]. Bulletin of Surveying and Mapping, 2016, 10: 62-64.

4. Wei Jialei. Research on geometric registration of UAV high-resolution images based on SIFT algorithm[J]. Beijing Surveying and Mapping, 2019, 33(01): 49-53.

5. Huang Haibo, Li Xiaoling, Xiong Wenyi. Overview of image registration based on SIFT algorithm [J]. Software Guide, 2019, 18(01): 1-4.

6. Wang Guo, Sha Congshu, Jiang Ruibo, et al. Open-pit mine engineering calculation based on drone tilt photography[J]. Metal Mine, 2017(10): 72-75.

7. Wang Guo, Jiang Ruibo, Xiao Honghai, et al. Threedimensional reconstruction of open-pit mine slope based on drone tilt photography[J]. China Mining, 2017, 26(4): 158-161.

8. Zhao Mandan, Wu Gaochang, Yan Shi, etc. Multiview camera joint calibration and improved PMVS reconstruction method [J]. Journal of Surveying and Mapping Science and Technology, 2017, 34(4): 387391.

9. Ba Boqing, Zhang Jifu, Xiao Linping, et al. Discussion on calculation method of earth-rock stripping engineering quantity in large open-pit coal mine[J]. Mine Survey, 2012(4): 65-68.

10. Zhang Yuxia, Lan Pengtao, Jin Yuanchun, et al. Practice and exploration of drone three-dimensional oblique photography technology in open-pit mine monitoring $[\mathrm{J}]$. Bulletin of Surveying and Mapping, 2017 (S1): 114-11 